\title{
Saúde das mulheres: vulnerabilidade, políticas de saúde e cuidado de enfermagem na pandemia de COVID-19
}

\author{
Women's health: vulnerability, health policies and nursing care in the COVID-19 pandemic \\ Salud de las mujeres: vulnerabilidad, políticas de salud y atención de enfermería en la pandemia
}

COVID-19

\author{
Mônica Maria de Jesus Silva \\ ORCID: https://orcid.org/0000-0002-4532-3992 \\ Universidade de São Paulo, Brasil \\ E-mail: monicamjs@usp.br \\ Angeline Lettiere-Viana \\ ORCID: https://orcid.org/0000-0002-4913-0370 \\ Universidade de São Paulo, Brasil \\ E-mail: angelina.lettiere@usp.br \\ Juliana Cristina dos Santos Monteiro \\ ORCID: https://orcid.org/0000-0001-6470-673X \\ Universidade de São Paulo, Brasil \\ E-mail: jumonte@eerp.usp.br \\ Flávia Azevedo Gomes-Sponholz \\ ORCID: https://orcid.org/0000-0003-1540-0659 \\ Universidade de São Paulo, Brasil \\ E-mail: flagomes@eerp.usp.br \\ Patrícia Scotini Freitas \\ ORCID: https://orcid.org/0000-0002-8270-8955 \\ Universidade Federal de Alfenas, Brasil \\ E-mail: patrícia.freitas@unifal-mg.edu.br \\ Maria José Clapis \\ ORCID: https://orcid.org/0000-0002-2896-3808 \\ Universidade de São Paulo, Brasil \\ E-mail: maclapis@eerp.usp.br
}

\begin{abstract}
Resumo
Este artigo apresenta uma reflexão sistematizada e discussão em torno de três eixos orientadores: o primeiro discute a vulnerabilidade das mulheres potencializada pela pandemia da COVID-19; no segundo, reflete sobre as políticas políticas públicas de saúde das mulheres no Brasil e estratégias de enfrentamento da COVID-19 com o objetivo de manter a saúde das mulheres e mitigar os efeitos da pandemia; e o terceiro reflete sobre o cuidado de enfermagem às mulheres em situação de vulnerabilidade frente a pandemia. A conclusão é que a pandemia da COVID-19 impactou sobremaneira as mulheres em situação de vulnerabilidade com consequências incontáveis e que adensam as iniquidades e as muitas vulnerabilidades já existentes. O cenário pós-pandemia exigirá esforços coletivos para proteger e garantir a saúde das mulheres em situação de vulnerabilidade, que estão entrelaçados à aticulação das políticas públicas com os procedimentos específicos das organizações de saúde; à participação de mulheres na formulação dessas políticas e ao cuidado de enfermagem.

Palavras-chave: Enfermagem; Cuidados de enfermagem; Políticas de saúde; Pandemias; Infecções por Coronavírus.
\end{abstract}

\begin{abstract}
This article presents a systematized reflection and discussion around three guiding axes: the first discusses the vulnerability of women enhanced by the COVID-19 pandemic; in the second, it reflects on public policies for women's health in Brazil and COVID-19's coping strategies with the objective of maintaining women's health and mitigating the effects of the pandemic; and the third reflects on the nursing care for women in vulnerable situations in the face of the pandemic. The conclusion is that the COVID-19 pandemic has had a major impact on vulnerable women with countless consequences that add to the inequities and the many vulnerabilities that already exist. The post-pandemic scenario will require collective efforts to protect and guarantee the health of women in vulnerable situations, which are intertwined with the articulation of public policies with the specific procedures of health organizations; the participation of women in the formulation of these policies and nursing care.
\end{abstract}

Keywords: Nursing; Nursing care; Health Policies; Pandemics; Coronavirus infections. 


\begin{abstract}
Resumen
Este artículo presenta una reflexión y discusión sistematizada en torno a tres ejes rectores: el primero discute la vulnerabilidad de las mujeres potenciadas por la pandemia COVID-19; en el segundo, reflexiona sobre las políticas públicas para la salud de la mujer en Brasil y las estrategias de afrontamiento del COVID-19 con el objetivo de mantener la salud de la mujer y mitigar los efectos de la pandemia; y el tercero reflexiona sobre la atención de enfermería a mujeres en situación de vulnerabilidad ante la pandemia. La conclusión es que la pandemia de COVID19 ha tenido un gran impacto en las mujeres vulnerables con innumerables consecuencias que se suman a las inequidades y las muchas vulnerabilidades que ya existen. El escenario pospandémico requerirá esfuerzos colectivos para proteger y garantizar la salud de las mujeres en situación de vulnerabilidad, los cuales se entrelazan con la articulación de las políticas públicas con los procedimientos específicos de las organizaciones de salud; la participación de las mujeres en la formulación de estas políticas y cuidados de enfermería.
\end{abstract}

Palabras clave: Enfermería; Cuidado de enfermería; Políticas de Salud; Pandemias; Infecciones por Coronavirus.

\title{
1. Introdução
}

A pandemia da COVID-19 ocasionada em decorrência da doença por Coronavírus - COVID-19, causada pelo SARSCoV-2, foi decretada pela Organização Mundial da Saúde (OMS) em março de 2020 (Liu et al., 2020). Tal situação, que se caracteriza como Emergência em Saúde Pública de Importância Internacional, fez com que a preocupação dos agentes sanitários e dos órgãos oficiais mundiais aumentasse, com especial atenção às populações mais vulneráveis.

No Brasil, até o dia 7 de julho de 2020 foram confirmados 18.909 .537 casos e 528.540 óbitos na população geral, segundo dados do Ministério da Saúde (Brasil, 2020). Somam-se a estes números, os alarmantes dados de óbitos maternos que já superaram o número notificado em 2020. De acordo com o Observatório Obstétrico Brasileiro COVID-19 (OOBr COVID19), ocorreram 454 óbitos em gestantes e puérperas por Covid-19 no país naquele ano. Até o dia 7 de julho de 2021, foram registrados 1063 óbitos nessa população, denotando um aumento preocupante (Rodrigues, Lacerda, \& Francisco, 2021).

Para além do número de casos e óbitos, a pandemia de COVID-19 promove consequências devastadoras e com diferentes impactos em contextos locais, uma vez que as iniquidades sociais e as vulnerabilidades já existentes em diversos grupos populacionais são potencializadas, como ocorre entre as mulheres. Isto decorre porque as mulheres sofrem de forma desproporcional os impactos das crises econômicas e sociais em virtude da estrutura patriarcal que mantem ativa a divisão sexual do trabalho, a qual se expressa na distribuição desigual das atividades de cuidado e organização doméstica. Tal desproporção exacerbou-se na pandemia da COVID-19 com o desencadeamento de uma convivência doméstica e familiar intensa em espaços e condições nem sempre adequados para atender as novas necessidades de trabalho remoto, ensino à distância e cuidados domésticos/familiares, evidenciando as desigualdades sociais, econômicas, o racismo e sexismo estruturais presentes na sociedade brasileira e a importância da consideração da perspectiva de gênero em todas as medidas para a contenção da COVID-19 (Organização das Nações Unidas, 2020).

Esse contexto estimula a reflexão sobre as desiguais formas de vivenciar a COVID-19 frente às vulnerabilidades as quais as mulheres brasileiras estão submetidas, bem como discutir as políticas de saúde e as estratégias de enfrentamento da COVID-19 que passarão a ser necessárias durante e a após a pandemia.

Assim, o objetivo é refletir sobre as diversas vulnerabilidades as quais as mulheres estão expostas e foram potencializadas pela pandemia da COVID-19; as políticas públicas de saúde das mulheres no Brasil e as estratégias de enfrentamento à COVID-19; bem como, o cuidado de enfermagem à essas mulheres frente a pandemia.

\section{Metodologia}

Reflexão teórica com abordagem metodológica fundamentada na análise e discussão de estudos e documentos publicados sobre as políticas públicas em saúde da mulher no Brasil e das recomendações sobre o novo Coronavírus.

O cenário da pandemia COVID-19 evidenciou a vulnerabilidade das mulheres com pontos de reflexão importantes a 
serem discutidos frente ao cuidado de enfermagem e as políticas públicas de saúde, os quais foram descritos em três categorias: 1) Vulnerabilidade das mulheres potencializadas pela pandemia da COVID-19; 2) Políticas públicas de saúde das mulheres no Brasil e as estratégias de enfrentamento à COVID-19 e 3) Cuidado de Enfermagem frente à vulnerabilidade das mulheres na pandemia de COVID-19.

\section{Resultados e Discussão}

Apresenta-se a seguir as categorias temáticas:

\section{1) Vulnerabilidade das mulheres potencializada pela pandemia da COVID-19}

Considera-se que as relações entre as pessoas em uma sociedade e seus equipamentos sociais definem o grau de vulnerabilidade dos indivíduos. Nesse sentido, é importante compreender a gama de situações e condições individuais e sociais que permeiam as vulnerabilidades concretas na vida cotidiana das pessoas. Essas situações evidenciam violações de direitos humanos, pois sugerem que trajetórias pessoais e psicossociais dependem de relações de poder que só podem ser compreendidas em termos de seus significados locais e contextos estruturais. Nessa perspectiva, contextos em que relações de poder estruturam desigualdades de classe social, de raça/cor, de gênero e orientação sexual podem ser analisados de forma interseccional, definindo trajetórias pessoais de vulnerabilidade (Do Carmo \& Guizardi, 2018).

Assim, a vulnerabilidade das mulheres está relacionada a situações que determinam os piores desfechos de saúde em cenários de iniquidades sociais, entendidas como desigualdades resultantes de injustiças e exclusão social como pobreza, exploração e abuso, aspectos psicossociais e culturais que incluem mulheres em condições socioeconômicas precárias, em situação de rua, em situação de violência, mulheres negras, indígenas, trabalhadoras da área rural, lésbicas, usuárias de drogas ou qualquer outra situação de discriminações e sofrimentos que afetam diretamente sua saúde e as condições de acesso à ela (França, Oliveira, \& Silva, 2016).

O avanço da pandemia de COVID-19 evidenciou que estas mulheres sofrem de modo adicional os seus impactos ao estarem imersas em estruturas desiguais que favorecem a disseminação do vírus e seus efeitos, ressaltando diferenças e iniquidades.

Neste sentido, cabe destacar o maior risco de morbimortalidade associada à COVID-19 entre mulheres no ciclo gravídico-puerperal, o que coloca gestantes e puérperas no grupo de risco segundo as recomendações dos órgãos de saúde. Adicionalmente, é imperativo mencionar os impactos desproporcionais entre mulheres negras, grávidas e puérperas, as quais apresentaram mortalidade materna quase duas vezes maior do que a observada em mulheres brancas na pandemia da COVID19. Esses indicadores evidenciam as disparidades raciais em saúde presentes no sistema de saúde, os desafios adicionais enfrentados pelas mulheres negras na pandemia e a necessidade da implementação de políticas públicas que avancem no combate aos determinantes sociais da saúde fora dos hospitais (Santos et., 2020).

A realidade do distanciamento social é desafiadora para as mulheres em situação de violência, principalmente para aquelas que vivem no mesmo ambiente que o agressor. Em decorrência disso, essas mulheres podem enfrentar obstáculos adicionais durante a pandemia de COVID-19 para acessar serviços essenciais de denúncia e rede de proteção, como sinalizado pela Organização das Nações Unidas (ONU) que evidencia que a violência doméstica triplicou na pandemia (Organização das Nações Unidas, 2020).

As mulheres em situação de rua, a julgar pelas condições em que vivem ou sobrevivem, podem ser consideradas como similares aos limites da pobreza e vulnerabilidade social e, na pandemia, por se encontrarem em ambientes de aglomeração formais, como albergues e casas de passagem, ou informais, como locais abandonados, estão mais susceptíveis à contaminação pelo vírus SARS-Cov-2 (Tai \& Wilson, 2020). 
A pandemia ainda evidenciou a situação das mulheres privadas de liberdade, as quais são provavelmente mais vulneráveis à COVID-19 do que a população em geral devido as condições de confinamento em que vivem por um período prolongado de tempo, aliado ao fato de possuírem uma carga subjacente maior de doenças, piores condições de saúde, maior exposição a riscos como tabagismo, e higiene e defesa imunológica deficientes (World Health Organization, 2020a).

No caso das mulheres que integram a população LGBTQIA+, somam-se aos impactos da pandemia promovidos pelas vulnerabilidades sociais, as consequências geradas pela LGBTIfobia que potencializa, modifica e altera a capacidade de proteção desse grupo em alguns eixos como as insuficientes formas de implementar medidas gerais de prevenção; as iniquidades em saúde preexistentes que podem ser potencializadas pela COVID-19 e as dificuldades no acesso aos serviços de saúde (Silva \& Melo, 2021).

Em relação às mulheres com deficiência, cabe ressaltar que enfrentam a falta de informações acessíveis sobre saúde pública e barreiras significativas para implementar medidas básicas de higiene, assim como falta de acesso a instalações de saúde. A COVID-19 agravou ainda mais essa situação, impactando desproporcionalmente as pessoas com deficiência, direta e indiretamente, caracterizando este grupo de quase um bilhão de pessoas como um dos mais atingidos pela pandemia (United Nations, 2020).

Diante destas situações e de muitas outras evidenciadas pela pandemia, globalmente, os governos estão criando respostas por meio de medidas de prevenção como a restrição social, recomendação quanto ao uso de máscaras e higienização das mãos com água e sabão ou álcool em gel. No entanto, essas medidas podem representar obstáculos substanciais para a assistência contínua às mulheres em situação de vulnerabilidade, desenhando um contexto desafiador no enfrentamento da pandemia aos profissionais de saúde, entre eles o enfermeiro, ao se considerar o acesso limitado dessa população à higienização, aos serviços de saúde e a ambientes que permitem o distanciamento físico e a restrição social.

Corroborando estas condições, organizações sociais e governamentais já relataram acesso reduzido de mulheres vulneráveis às medidas de prevenção e proteção à pandemia. A Organização Pan-Americana de Saúde (OPAS) enfatizou a necessidade de uma resposta global e coordenada à COVID-19 que atenda às necessidades específicas das populações vulneráveis e instigou os países a promoverem medidas de proteção a essa população (Organização Pan-Americana de Saúde, 2020).

Diante do exposto, torna-se notório o grande desafio de reduzir as vulnerabilidades das mulheres e mais ainda, tona-se evidente, urgente e necessário a articulação de políticas públicas com os procedimentos específicos das organizações de saúde para protege-las da COVID-19, com enfoque nas estratégias de enfrentamento, que será o tópico discutido a seguir.

\section{2) Políticas públicas de saúde das mulheres no Brasil e estratégias de enfrentamento da COVID-19}

Ao longo dos últimos 20 anos, inúmeras políticas públicas foram elaboradas no Brasil para promover a equidade em saúde e atender as necessidades das mulheres em situação de vulnerabilidade. Entre elas, cabe destacar a Política Nacional de Atenção Integral à Saúde da Mulher (PNAISM) que foi implementada em 2004 e estabeleceu, entre seus objetivos específicos, a promoção da saúde da mulher em situação de violência doméstica e sexual, das mulheres negras, indígenas, residentes e trabalhadoras da área rural e a saúde da mulher em situação de prisão, como grupos prioritários em virtude das vulnerabilidades associadas às especificidades destes grupos de mulheres (Brasil, 2004).

Apesar da expansão e dos avanços alcançados por essa política, nos últimos anos ocorreram inúmeros retrocessos em virtude de um modelo conservador imposto pelo atual Governo Federal. Além disso, no cenário brasileiro, é preciso reconhecer as disparidades regionais das redes de atenção à saúde, as quais podem ser frágeis e fragmentadas para enfrentar uma emergência como a COVID-19.

Especificamente em relação à mulher em situação de violência, foi criada a Política Nacional de Enfrentamento à 
Violência contra as Mulheres cujo objetivo é enfrentar todas as formas de violência contra as mulheres a partir de uma perspectiva de gênero e de uma visão integral deste fenômeno, o que inclui a sua saúde (Brasil, 2011).

A Política de Saúde Integral de Lésbicas, Gays, Bissexuais, Travestis e Transexuais foi implementada com o objetivo promover a saúde integral dessa população, eliminando a discriminação e o preconceito institucional e contribuindo para a redução das desigualdades e para consolidação do SUS como sistema universal, integral e equitativo, abrangendo mulheres que historicamente sofrem preconceito e discriminação em virtude de suas sexualidades e gêneros se localizarem fora do modelo cisgênero e heterossexual, o que demonstra a manutenção das hierarquias sociais nas quais elas estão inseridas (Silva \& Melo, 2021, Brasil, 2013).

Quanto às mulheres em privação de liberdade, a portaria interministerial $n^{\circ} 1$, de 2 de janeiro de 2014 instituiu a Política Nacional de Atenção Integral à Saúde das Pessoas Privadas de Liberdade no Sistema Prisional (PNAISP) no âmbito do SUS, a qual possui entre seus princípios a integralidade da atenção à saúde da população privada de liberdade no conjunto de ações de promoção, proteção, prevenção, assistência, recuperação e vigilância em saúde, executadas nos diferentes níveis de atenção (Brasil, 2014).

A Política Nacional de Saúde da Pessoa com Deficiência, que abrange as mulheres nesta condição, foi instituída em 2002, visando a inclusão das pessoas com deficiência em toda a rede de serviços do SUS (Brasil, 2002).

No que se refere as mulheres em situação de rua, o Decreto n 7.053 de 23 de dezembro de 2009 instituiu a Política Nacional para a População em Situação de Rua (PSR) (Brasil, 2009).

Estas três últimas políticas mesmo não sendo específicas da saúde, cabem ser destacadas, uma vez que tem o objetivo de assegurar condições para o acesso amplo, simplificado e seguro aos serviços e programas que integram as políticas públicas de saúde às mulheres nestas condições.

Embora as políticas públicas brasileiras mencionadas tenham sido formuladas visando abranger as mulheres em sua totalidade e diversidade, promover a equidade em saúde e atender as necessidades das mulheres em situação de vulnerabilidade, várias delas sofreram retrocessos nos últimos anos. Ademais, a pandemia da COVID-19 exacerbou fragilidades em vários âmbitos de tais políticas, que são exemplares em sua formulação, mas que enfrentam inúmeros obstáculos em sua real implementação.

Nesse contexto, ao se discutir a prevenção e o enfrentamento de diversos grupos contra a pandemia da COVID-19, há significativas diferenças que devem ser consideradas principalmente quando se faz presente realidades permeadas por vulnerabilidades já existentes.

Dessa forma, para que se tenham avanços na consolidação da saúde das mulheres em situação de vulnerabilidade, urge a necessidade de articulações que possibilitem a implementação efetiva de políticas públicas e de reconhecimento de que as práticas inclusas nas políticas estão entrelaçadas com as consequências sociais que determinam as vulnerabilidades e influenciam as respostas aos problemas, como a atual pandemia.

Desde o início da mesma, diversos protocolos e recomendações foram publicados na esfera da saúde das mulheres e em atenção às diversas situações de vulnerabilidade que permeiam essa população.

Neste âmbito, a OMS lançou em 17 de abril de 2020 a recomendação "Continuação dos serviços essenciais de saúde sexual, reprodutiva, materna, neonatal, infantil e adolescente durante a pandemia COVID-19” (World Health Organization, 2020b).

No cenário brasileiro, várias Notas Técnicas federais e estaduais também foram lançadas nos últimos meses para atender a necessidade de mulheres no ciclo gravídico-puerperal, inclusive as incluindo no grupo de risco para COVID-19, na medida em que novas descobertas sobre a doença foram publicadas. Com relação à enfermagem, a Associação Brasileira de Obstetrizes e Enfermeiros Obstetras (ABENFO) e a Sociedade Brasileira de Enfermeiros Pediatras (SOBEP) lançaram em abril 
de 2020 a "Nota Técnica referente aos cuidados da equipe de enfermagem obstétrica, neonatal e pediátrica diante de caso suspeito ou confirmado de COVID-19”, com orientações para guiar as equipes de enfermagem que atuam na assistência obstétrica, neonatal e pediátrica em serviços públicos ou privados durante a pandemia (Associação Brasileira de Enfermeiros Obstetras, 2020).

Relativo as mulheres em privação de liberdade, é importante mencionar o guia "Preparação, prevenção e controle da COVID-19 em prisões e outros locais de detenção, lançado pela OMS em 15 de março de 2020, com recomendações para evitar ou minimizar a ocorrência da infecção e de surtos graves nas prisões e locais de detenção (World Health Organization, 2020a).

Também merece destaque o documento "Sumário de Políticas: Uma resposta inclusiva para as pessoas com deficiência face à pandemia por COVID-19”, lançado pela ONU com recomendações para tornar a resposta e a recuperação inclusivas para as pessoas com deficiência (United Nations, 2020).

Essas recomendações implementadas por líderes, governantes, associações científicas e organizações de saúde nacionais e internacionais tem como objetivo controlar e mitigar a transmissão, a redução do número de casos e a diminuição de internações nas unidades hospitalares, evitando a disseminação do vírus e a morte de mulheres. No entanto, a pandemia trouxe à tona um novo panorama de incertezas global, ressaltadas no contexto local por marcadores sociais que não atuam de forma isolada e amplificam a vulnerabilização estrutural que permeia a realidade vivenciada pelas mulheres brasileiras, o que inviabiliza a implementação de medidas eficazes de prevenção e enfrentamento da COVID-19 à muitos subgrupos dessa população.

Outrossim, há de se refletir sobre o cenário pós-pandemia e reconhecer que medidas de enfrentamento à COVID-19 precisarão ser implementadas no Brasil por um longo tempo, o que exigirá esforços coletivos para proteger e garantir a sobrevivência das mulheres em situação de vulnerabilidade.

Essa reflexão leva a percepção da importância da participação de mulheres na formulação das políticas públicas de saúde. Sem equilíbrio de gênero nas posições de liderança, as necessidades específicas das mulheres podem não ser priorizadas e não incluídas na tomada de decisões ou no desenho de respostas, acarretando políticas de gênero neutras ou mesmo práticas discriminatórias de gênero. Além disso, onde existem políticas equitativas de gênero é comum a falta de implementação ou aplicação efetiva (Wenhem, Smith \& Morgan, 2020).

Não menos importante, considerando-se que a força de trabalho da linha de frente da pandemia da COVID-19 é composta principalmente por mulheres, suas vozes e necessidades devem ser adequadamente representadas e priorizadas nas políticas organizacionais e no planejamento de respostas à pandemia. Para tanto, infere-se a necessidade da promoção de padrões e cultura profissionais que garantam que as ideias e necessidades específicas das mulheres sejam consideradas, promova a sua liderança e a participação equitativa na tomada de decisões.

Frente a estas compreensões, ratifica-se a importância das políticas públicas no que diz respeito à consolidação de ações direcionadas ao enfrentamento dos problemas e das necessidades apresentadas pelas mulheres em situação de vulnerabilidade, para o planejamento e tomada de decisão na atuação do enfermeiro.

\section{3) Cuidado de enfermagem frente à vulnerabilidade das mulheres na pandemia de COVID-19}

No cenário da pandemia da COVID-19 fica evidente que o mundo passou por um momento de transformação que exigiu um novo olhar para o cuidado em saúde e em enfermagem, demonstrando a necessidade de adequação do processo de organização e implementação de políticas públicas efetivas que sustentem o enfrentamento da vulnerabilidade das mulheres como estratégia de proteção da saúde e, consequentemente, de oferta de cuidado integral às mulheres em situações de emergência sanitária. 
Frente a esta realidade, globalmente, enfermeiros e parteiras têm trabalhado continuamente e intensamente na pandemia da COVID-19, salvando vidas, proporcionando conforto diante da morte, promovendo a educação em saúde sobre medidas de proteção para impedir a propagação viral, cuidando dos que não estão infectados com o vírus e gerando evidências científicas por meio de pesquisas que podem colaborar na tomada de decisões no atendimento à população afetada (Turale, Meechamnan \& Kunaviktikul, 2020).

No enfrentamento da situação de vulnerabilidade, o cuidado de enfermagem não se restringe apenas à questão do combate à mesma, mas compreende também as dimensões da prevenção, da assistência e da garantia de direitos, os quais são, muitas vezes, permeados por obstáculos e desafios inerentes a não implementação ou a implementação inadequada das políticas públicas em saúde.

A manutenção do cuidado de enfermagem à saúde é essencial para reduzir as mortes pela COVID-19, bem como os efeitos adversos à saúde entre as mulheres em situação de vulnerabilidade, o que exige entender e priorizar suas necessidades de saúde, além de garantir o acesso aos serviços de saúde e a segurança, incluindo as mulheres trabalhadoras na linha de frente. Isto incita a reflexão sobre as práticas de enfermagem e seu processo de trabalho que deve contribuir para o alcance do cuidado integral à mulher, entendido como aquele que atende às múltiplas dimensões do ser humano considerando os aspectos culturais, sociais, econômicos, religiosos, afetivos e biológicos.

Tais reflexões emergem a importância do trabalho do enfermeiro no enfrentamento da vulnerabilidade das mulheres no contexto da pandemia da COVID-19 e reiteram sua essencialidade, que muitas vezes, se faz ausente dos espaços de decisão e encontra dificuldades no campo da autonomia, o que culmina com a ausência de um real reconhecimento do seu papel para a organização dos serviços de saúde.

Este estudo traz contribuições para a enfermagem ao fornecer elementos para a reflexão dos enfermeiros quanto ao agravamento da vulnerabilidade da mulher gerado no contexto da pandemia da COVID-19, auxiliando-os no exercício e planejamento do cuidado, na elaboração de políticas públicas, na implementação de medidas de enfrentamento a situações de emergência mais inclusivas e na formação profissional.

\section{Considerações Finais}

A pandemia impactou sobremaneira as mulheres em situação de vulnerabilidade com consequências incontáveis e que adensam as iniquidades e as muitas vulnerabilidades já existentes. As recomendações ao novo coronavírus, nacionais e internacionais, sinalizam uma evidente e necessária preocupação sobre a situação de vulnerabilidade das mulheres.

O cenário pós-pandemia exigirá esforços coletivos para proteger e garantir a saúde das mulheres em situação de vulnerabilidade, que estão entrelaçados à articulação das políticas públicas com os procedimentos específicos das organizações de saúde; à participação de mulheres na formulação dessas políticas e à essencialidade do cuidado de enfermagem.

\section{Referências}

Associação Brasileira de Obstetrizes e Enfermeiros Obstetras (ABENFO) \& Sociedade Brasileira de Enfermeiros Pediatras (SOBEP) (2020). Nota Técnica referente aos cuidados da equipe de Enfermagem Obstétrica, Neonatal e Pediátrica diante de caso suspeito ou confirmado de COVID-19. https://sobep.org.br/covid-19-nota-tecnica-referente-aos-cuidados-da-equipe-de-enfermagem-obstetrica-neonatal-e-pediatrica-diante-de-caso-suspeito-ouconfirmado/

Brasil (2002). Ministério da Saúde. Gabinete do Ministro. Portaria $n^{o}$ 1060, de 5 de junho de 2002. Aprova, na forma do Anexo desta Portaria, a Política Nacional de Saúde da Pessoa Portadora de Deficiência. http://bvsms.saude.gov.br/bvs/saudelegis/gm/2002/prt1060_05_06_2002.html

Brasil (2004). Ministério da Saúde. Política Nacional de Atenção Integral à Saúde da Mulher: princípios e diretrizes. https://bvsms.saude.gov.br/bvs/publicacoes/politica_nac_atencao_mulher.pdf

Brasil (2009). Presidência da República. Casa Civil. Decreto $n^{o} 7.053$ DE 23 de dezembro de 2009. Institui a Política Nacional para a População em Situação de Rua e seu Comitê Intersetorial de Acompanhamento e Monitoramento, e dá outras providências. http://www.planalto.gov.br/ccivil_03/_ato20072010/2009/decreto/d7053.htm 
Brasil (2013). Ministério da Saúde. Política Nacional de Saúde Integral de Lésbicas, Gays, Bissexuais, Travestis e Transexuais. https://bvsms.saude.gov.br/bvs/publicacoes/politica_nacional_saude_lesbicas_gays.pdf

Brasil (2014). Ministério da Saúde. Diário Oficial da União. Portaria interministerial $n^{\circ} 1$, de 2 de janeiro de 2014. Institui a Política Nacional de Atenção Integral à Saúde das Pessoas Privadas de Liberdade no Sistema Prisional (PNAISP) no âmbito do Sistema Único de Saúde (SUS). http://bvsms.saude.gov.br/bvs/saudelegis/gm/2014/pri0001_02_01_2014.html

Brasil (2011). Presidência da República. Secretaria de Políticas para as Mulheres. Política Nacional de Enfrentamento à Violência Contra as Mulheres. https://www12.senado.leg.br/institucional/omv/entenda-a-violencia/pdfs/politica-nacional-de-enfrentamento-a-violencia-contra-as-mulheres

Brasil. (2020). Ministério da Saúde. Covid-19 no Brasil. https://qsprod.saude.gov.br/extensions/covid-19_html/covid-19_html.html. Acesso em 08/07/2021

Do Carmo, M. E. \& Guizardi, F. L. (2018). The concept of vulnerability and its meanings for public policies in health and social welfare. Cad. Saúde Pública (Online), 34(3), e00101417.

França, A. M. B. \& Oliveira e Silva J. M. (2016). Women in vulnerable situation: a view from perspective of social phenomenology. Rev. Enferm. UFPE on line, 10(supl 2), 875-9.

Li, Q. et al. (2020). Early transmission dynamics in Wuhan, China, of novel coronavirus-infected pneumonia. N Engl J Med, 382(13), $1199-207$.

Organização das Nações Unidas (2020). Diretrizes para atendimento em casos de violência de gênero contra meninas e mulheres em tempos da pandemia da COVID-19. https://www.onumulheres.org.br/wp-content/uploads/2020/08/Diretrizes-para-atendimento_ONUMULHERES.pdf

Organização Panamericana de Saúde. (2020). OPAS pede que países protejam grupos vulneráveis dos efeitos da pandemia. https://www.paho.org/bra/index.php?option=com_content\&view=article\&id=6175:diretora-da-opas-pede-que-paises-protejam-grupos-vulneraveis-dos-efeitosda-pandemia-de-covid-19\&Itemid=812

Rodrigues, A., Lacerda, L., \& Francisco, R. P. V. (2021). Brazilian Obstetric Observatory. 8 Jul 2021. https://observatorioobstetrico.shinyapps.io/covid_gesta_puerp_br/

Santos, D. S. et al. (2020). Disproportionate impact of COVID-19 among pregnant and postpartum Black Women in Brazil through structural racism lens. Clin Infect Dis., ahead of print,1-9.

Silva, A. D. \& Melo, S. L. P. (2021). Pandemia de covid-19 e população LGBTI+. (In)visibilidades dos impactos sociais. Sex., Salud Soc, 37 , e-21202.

Tsai, J. \& Wilson, M. (2020). COVID-19: a potential public health problem for homeless populations. Lancet Public Health,5(4), e186-e187.

Turale, S., Meechamnan, C., \& Kunaviktikul, W. (2020). Challenging Times: Ethics, Nursing and the COVID-19 Pandemic. Int Nurs Rev., 67(2), 164-7.

United Nations (2020). Policy brief: a disability-inclusive response to COVID-19. https://unsdg.un.org/sites/default/files/2020-05/Policy-Brief-A-DisabilityInclusive-Response-to-COVID-19.pdf

Wenham, C., Smith, J. \& Morgan, R. (2020). COVID-19: the gendered impacts of the outbreak. Lancet, 395(1027), 846-8.

World Health Organization. (2020a). Preparedness, prevention and control of COVID-19 in prisons and other places of detention: Interim guidance. https://apps.who.int/iris/bitstream/handle/10665/336525/WHO-EURO-2020-1405-41155-55954-eng.pdf?sequence=1\&isAllowed=y

World Health Organization (2020b). Regional Office for South-East Asia. Continuing essential Sexual Reproductive, Maternal, Neonatal, Child and Adolescent Health services during COVID-19 pandemic. https://www.who.int/pmnch/media/news/2020/SRMNCAH.pdf?ua=1 